\title{
Management of Cognitive Dysfunction
}

\author{
J.E. Morley \\ Division of Geriatric Medicine, Saint Louis University School of Medicine, St. Louis, Missouri
}

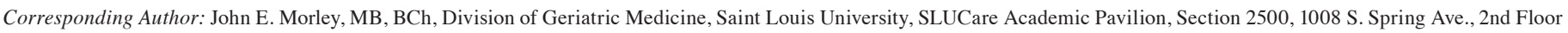
St. Louis, MO 63110,Email: john.morley@health.slu.edu, Twitter: @drjohnmorley

Key words: Cognitive dysfunction, elderly, screening, aging brain.

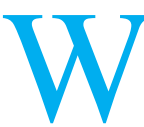
ith increasing age cognitive dysfunction becomes very common (1). Older persons with cognitive dysfunction commonly engage in unsafe activities such as poor medication management, driving, and going to their physician alone (2). For this reason, it is important that the primary care physician annually screen persons over 65 years for cognitive dysfunction. The MiniMental Status Examination (MMSE) has been the classical screening test but now requires payment if it is used. Both the Montreal Cognitive Assessment (MOCA) and the St. Louis University Mental Status (SLUMS) examination are more sensitive and specific than the MMSE $(3,4)$. The MOCA now requires health professionals to pay to be trained to use it and is no longer in the free public domain (5). The SLUMS is free making it the test of choice. However, it takes 6 to 7 minutes to administer. That is too long for the average primary care health professional to administer. The MiniCog and the Rapid Cognitive Screen (RCS) which is derived from the SLUMS are both quicker to administer. The SLUMS can identify people with mild cognitive impairment as well as dementia, making it preferable to the MiniCog $(6,7)$.

The first of the nootropics introduced to treat dementia was the ergotoxine derivative, hydergine. A meta-analysis found that hydergine significantly improved cognition based on global ratings (8). However, these effects were considered to be modest.

Tacrine was the first cholinesterase inhibitor to be approved by the FDA in 1993. Its approval was withdrawn from the market in 2013 due to side effects. Cholinesterase inhibitors reduce the loss of brain function to a small degree in persons with Alzheimer's disease. Most of the studies are of 6 months or less and improve cognition in approximately 14 out of 100 persons (10). A 5-year study suggested that cholinesterase inhibitors increased the MMSE score by 0.13 points over this time period (11). Up to $30 \%$ of patients have side effects. Memantine, an N-methyl-D-aspartate receptor antagonist, together with donepezil improved cognition and global assessment but had more side effects than memantine alone (12). Aducanumab is a monoclonal antibody directed at betaamyloid that has received accelerated approval to treat early stage Alzheimer's disease (13). The preliminary data for this Received June 22, 2021 drug is at best questionable.

Recently, our group has shown in an epidemiological study that the tetanus, diphtheria, pertussis (Tdap) vaccine markedly reduces dementia in persons receiving it compared to unvaccinated individuals (14). This may be because of activation of regulatory $\mathrm{T}$ cells. This effect is much greater than seen with the available drugs for the treatment of dementia.

\section{Table 1. The Treatable Causes of Dementia}

Drugs - anticholinergic
Emotional - depression
Metabolic - hypothyroid, B12 deficiency
Ears/eyes deficits
Normal pressure hydrocephalus
Tumors
Infection
Atrial fibrillation
Sleep apnea

Perhaps more importantly there are many treatable causes of dementia. These are easily remembered by the mnemonic - DEMENTIAS (Table 1) (15). Sleep apnea is very common cause of cognitive dysfunction that needs to be recognized and treated (16). Other common treatable causes of dementia include atrial fibrillation, depression, anticholinergic drugs and decreased hearing. Metformin in persons with Type 2 diabetes mellitus reduces dementia $(17,18)$. The prevalence of dementia has been reduced in the USA over the last decade, most probably due to the treatment of hypertension $(19,20)$.

Most importantly, there are a number of nutritional and psychological interventions that appear to reduce dementia (Table 2). These include especially fruit and vegetable diets such as the Mediterranean (21) or the MIND diets (22). Extra virgin olive oil further decreases the chance of developing cognitive dysfunction by reducing inflammatory damage in the brain $(23,24)$. There is some evidence that yogurt $(25)$ and curcumin (26) may also decrease the development of cognitive dysfunction. Oligomannate, which is derived from seaweed, decreases microbiome inflammatory response similar to yogurt and improves cognition (27). 
Table 2. Nondrug Treatment of Dementia

\begin{tabular}{|l|l|}
\hline 1. & Manage treatable causes \\
2. & Mediterranean or MIND diet \\
3. & Extra virgin olive oil \\
4. & Yogurt/curcumin \\
5. & Exercise \\
6. & Cognitive Stimulation Therapy (CST) \\
7. & Reminiscence therapy \\
\hline 8. & Caregiving support \\
\hline
\end{tabular}

Multiple studies have shown that exercise improves memory (28). The FINGER study showed that a two-year study of Mediterranean diet, exercise, cognitive training and treatment of vascular risk factors compared to a control group resulted in improved executive function and processing speed (29). Cognitive Stimulation Therapy (CST) improves memory as well as available drugs $(30,31)$ and better in combination with exercise (32). Reminiscence therapy is less effective at improving memory (33). Finally, it should be recognized that high quality caregiving is a key component to maintaining the quality of life of persons with dementia and their family members (34).

Without medications to improve memory it is clear that there are multiple things a health care professional can do to improve cognition. Sadly, most of these are never done. Before health care professionals rush to utilize cognitive enhancers, it is essential that they develop high quality cognitive enhancement programs as outlined in Table 2 . It is important to recognize that in geriatric care there is often multiple approaches to enhancing outcomes that do not involve medications.

Conflicts of interest: The author declares there are no conflicts.

\section{References}

1. Sanford AM, Morley JE, Berg-Weger M, et al. High prevalence of geriatric syndromes in older adults. PLoS One 2020;Jun 5;15(6):e0233857. eCollection 2020.

2. Amjad H, Roth DL, Samus QM, et al. Potentially unsafe activities and living conditions of older adults with dementia. J Am Geriatr Soc 2016; Jun;64(6):12231232.

3. Suda S, Muraga K, Ishiwata A, et al. Early cognitive assessment following acute stroke: Feasibility and comparison between mini-mental state examination and Montreal Cognitive Assessment. J Stroke Ceerebrovasc Dis 2020 Apr;29(4):104688. Doi: 10.1016/j.jstrokecerebrovasdis.2020.104688. Epub 2020 Feb 14

4. Tariq SH, Tumosa N, Chibnall JT, et al. Comparison of the Saint Louis University mental status examination and the mini-mental state examination for detecting dementia and mild neurocognitive disorder-a pilot study. Am J Geriatr Psychiatry 2006 Nov;14(11):900-910.

5. Mansoor D, Erten-Lyons D. I have to pay to use the Montreal Cognitive Assessment: What should I do? (perspective). J Acad Consult Liaison Psychiatry 2021;62(2):253255 .

6. Borson S, Scanlan JM, Chen P, Ganguli M. The Mini-Cog as a screen for dementia: Validation in a population-based sample. J Am Geriatr Soc 2003;51:1451-1454.

7. Malmstrom TK, Voss VB, Cruz-Oliver DM, et al. The rapid cognitive screen (RCS): A point-of-care screening for dementia and mild cognitive impairment. J Nutr Health Aging 2015; 19:741-744.

8. Schneider L, Olin JT, Novit A, Luczak S. Hydergine for dementia. Cochrane Database of Systematic Reviews 2010;Issue 3. DOI: 10.1002/14651858.CD000359.

9. Ebrahem AS, Oremus M. A pharmacoeconomic evaluation of cholinesterase inhibitors and memantine for the treatment of Alzheimer's disease. Expert Opin Pharmacother $2018 ; 19: 1245-1259$.
10. Cui CC, Sun Y, Wang XY, Zhang Y, Xing Y. The effect of antidementia drugs on Alzheimer disease-induced cognitive impairment: A network meta-analysis. Medicine (Baltimore):2019 Jul;98(27):e16091. doi: 10.1097/MD.0000000000016091

11. Xu H, Garcia-Ptacek S, Jonsson L, et al. Long-term effects of cholinesterase inhibitors on cognitive decline and mortality. Neurology. 2021 Apr 27;96(17):e2220-e2230.

12. Guo J, Wang Z, Liu R, et al. Memantine, donepezil, or combination therapy - What is the best therapy for Alzheimer's Disease? A network meta-analysis. Brain Behav 2020;10(11):e01831. Epub Sep 10

13. Mullard A. Landmark Alzheimer's drug approval confounds research community. Nature 2021 Jun;594(7863):309-310.

14. Scherrer JF, Salas J, Wiemken TL, et al. Lower risk for dementia following adult tetanus, diphtheria and pertussis (Tdap) vaccination. J Gerontol A Biol Sci Med Sci 2021;Apr 15 DOI: 10.1093/gterona/glab115. Epub ahead of print.

15. Morley JE, Farr SA, Nguyen AD. Alzheimer Disease. Clin Geriatric Med 2018;34:591-601.

16. Morley JE, Sanford A, Bourey R. Sleep apnea: A geriatric syndrome. J Am Med Dir Assoc 2017;18(11):899-904.

17. Morley JE. Diabetes: The diabetic brain. Nat Rev Endocrinol 2017;13(10):570-571.

18. Scherrer JF, Salas J, Floyd JS, et al. Metformin and sulfonylurea use and risk of incident dementia. Mayo Clin Proc 2019;94:1444-1456.

19. Tom SE, Phadke M, Hubbard RA, et al. Association of demographic and early-life socioeconomic factors by birth cohort with dementia incidence among US adults born between 1893 and 1949. JAMA Network 2020;3(7):e2011094.

20. Knopman DS. The enigma of decreasing dementia incidence. JAMA Netw Open 2020 Jul 1;3(7):e2011199. Doi: 10.1001/jamanetworkopen.2020.11199.

21. Singh B, Parsaik AK, Mielke MM, et al. Association of Mediterranean diet with mild cognitive impairment and Alzheimer's disease: A systematic review and meta-analysis. J Alzheimers Dis 2014;39(2):271-282.

22. Morris MC, Tangney CC, Wang Y, et al. MIND diet slows cognitive decline with aging. Alzheimers Dement 2015;11:1015-1022.

23. Muñoz-Garcia MI, Martinez-González MA, Razquin C, et al. Exploratory dietary patterns and cognitive function in the "Seguimiento Universidad de Navarra" (SUN) Prospective cohort. Eur J Clin Nutr 2021 May 13. DOI: 10.1038/s41430-021-00922-5. Epub ahead of print.

24. Farr SA, Price TO, Dominguez LJ, et al. Extra virgin olive oil improves learning and memory in SAMP8 mice. J Alzheimers Dis 2012;28:81-92.

25. Beauchet $\mathrm{O}$, Launay $\mathrm{CP}$, Galery $\mathrm{K}$, et al. Effects of vitamin D and calcium fortified yogurts on gait, cognitive performances, and serum 25-hydroxyvitamin $D$ concentrations in older community-dwelling females: Results from the Gait, Memory, Dietary and Vitamin D (GAME-D2) randomized controlled trial. Nutrients. 2019; Nov 26:11(12):2880.

26. Small GW, Siddarth P, Li Z, et al. Memory and brain amyloid and tau effects of a bioavailable form of curcumin in non-demented adults: A double-blind, placebocontrolled 18-month trial. Am J Geriatr Psychiatry 2018;26:266-277.

27. Xiao S, Chan P, Wang T, et al. A 36-week multicenter, randomized, double-blind, placebo-controlled, parallel-group, phase 3 clinical trial of sodium oligomannate for mild-to-moderate Alzheimer's dementia. Alzheimers Res Ther 2021 Mar 17;13(1):62. Doi: 10.1186/s13195-021-00795-7.

28. Huang X, Zhao X, Li B, et al. Comparative efficacy of various exercise interventions in cognitive function in patients with mild cognitive impairment or dementia: A systematic review and network meta-analysis. J Sport Health Sci 2021;16:S20952546(21)00051-X.

29. Ngandu T, Lehtisalo J, Solomon A, et al. A 2 year multidomain intervention of diet, exercise, cognitive training, and vascular risk monitoring versus control to prevent cognitive decline in at-risk elderly people (FINGER): A randomized controlled trial. Lancet 2015 Jun 6;385(9984):2255-2263.

30. Woody B, Aguirre E, Spector AE, Orrell M. Cognitive stimulation to improve cognitive functioning in people with dementia. Cochrane Database Syst Rev $2012 \mathrm{Feb}$ 15;(2):CD005562.

31. Holden E, Stoner CR, Spector A. Cognitive stimulation therapy for dementia: Provision in National Health Service settings in England, Scotland and Wales Dementia (London) 2020 Sep 7; DOI: 10.1177/1471301220954611.

32. Lundy J, Hayden D, Pyland S, Berg-Seger M, Malmstrom TK, Morley JE. An agefriendly health system. J Am Geriatr Soc 2021 Mar;69(3):806-812.

33. Woods B, O'Philbin L, Farrell EM, Spector AE, Orrell M. Reminiscence therapy for dementia. Cochrane Database Syst Rev 2018 Mar 1;3(3):CD001120.

34. Larson EB, Stroud C. Meeting the challenge of caring for persons living with dementia and their care partners and caregivers: A report from the National Academies of Sciences, Engineering, and Medicine. National Academies Press (US): 2021 Feb 23. ISBN-13:978-0-309-15429-1

How to cite this article: J.E. Morley. Management of Cognitive Dysfunction. J Nutr Health Aging. 2021;25(7):819-820; http:/ / dx.doi.org/10.1007/s12603-021-1654-y 